\title{
The Predictors of Long-Term Hospitalization in Turkish Heart Failure Population: A Subgroup Analysis of Journey Heart Failure-TR Study: On Behalf of Journey Heart Failure-TR Investigators
}

\author{
Umit Yasar Sinan, Mehmet Erturk', Erkan Yıldırım², Duygu Koçyiğit ${ }^{3}$, Ilgın Karaca ${ }^{4}$, Faruk Ertas ${ }^{5}$, Ahmet Celik ${ }^{6}$, Fatih Aksoy ${ }^{7}$, Hasan Ali Gumrukcuoglu ${ }^{8}$,
} Umit Yuksek ${ }^{9}$, Mahir Cengiz ${ }^{10}$, Mehdi Zoghi ${ }^{11}$

Department of Cardiology, Istanbul University Institute of Cardiology, ${ }^{1}$ Department of Cardiology, Ministry of Health Mehmet Akif Ersoy Thoracic and Cardiovascular Surgery Training Research Hospital, Istanbul, ${ }^{2}$ Department of Cardiology, Health Science University Erzurum Region Education and Research Hospital, Erzurum, ${ }^{3}$ Department of Cardiology, Hacettepe University School of Medicine, Ankara, ${ }^{4}$ Department of Cardiology, Firat University School of Medicine, Elazig, ${ }^{5}$ Department of Cardiology, Dicle University School of Medicine, Diyarbakir, ${ }^{6}$ Department of Cardiology, Mersin University School of Medicine, Mersin, ${ }^{7}$ Department of Cardiology, Afyonkarahisar Dinar State Hospital, Afyonkarahisar, ${ }^{8}$ Department of Cardiology, Lokman Hekim Van Hospital, Van, ${ }^{9}$ Department of Cardiology, Izmir Odemis State Hospital, Izmir, ${ }^{10}$ Department of Cardiology, Istanbul University Cerrahpasa School of Medicine, Istanbul, ${ }^{11}$ Department of Cardiology, Ege University School of Medicine, Izmir, Turkey

\section{Abstract}

Background: Heart failure (HF) is an important public health problem. We aimed to investigate the predictors of long-term hospitalization in Turkish HF population. Materials and Methods: Journey-HF-TR study is a multicenter, cross-sectional, noninvasive, and observational study that was conducted in intensive care unit (ICU), coronary care unit (CCU), and cardiology wards in seven geographical regions of Turkey. In this subgroup analysis, patients were classified as two groups according to inhospital stay called the patient with the shorter length of stay (S-LOS) (inhospital stay $<5$ days; S-LOS) and patients with longer LOS (L-LOS) (inhospital stay $\geq 5$ days; L LOS). Results: The study group was consisted of 1606 patients (57.2\% male, mean age: $67.8 \pm 13.0$ years old). One thousand and thirty seven patients, whom in-hospital stay duration were recorded in case report form, were included in this analysis. There were 487 patients (32.1\%) in S LOS group and 1030 patients (67.9\%) in L LOS group. In multivariate analysis, correlation was present for NYHA functional capacity, CKD, ACS related HF, right HF, cardiogenic shock, invasive and noninvasive ventilation, and hemodynamic monetarization. The longer inhospital stay increases the probability of morbidity and mortality. Conclusion: We demonstrated that there was positive correlation between longer hospital stay and HF severity (NYHA III-IV), CKD, cardiogenic shock, right ventricular HF, and HF related to ACS. HFpEF patients have less in-hospital stay than HFrEF and HFmrEF patients.

Keywords: Acute heart failure, long inhospital stay (length of stay), morbidity, mortality

\section{INTRODUCTION}

Acute heart failure (AHF) is a life-threatening syndrome that constitutes a majority of the hospitalizations in cardiology clinics and intensive/coronary care units (CCUs) among patients aged above $65 .{ }^{[1]}$ It can develop as a result of worsening symptoms of existing HF (acute decompensated chronic HF (ADCHF) or de novo development of these symptoms. As well as, it is a leading cause of morbidity and mortality, and it also increases the health-care costs.

\begin{tabular}{|l|l|}
\hline \multicolumn{3}{c|}{ Access this article online } \\
\hline Quick Response Code: & Website: \\
& \\
http://www.ijcva.com
\end{tabular}

Hospital length of stay (LOS) is defined as the actual number of days the patients remained in the hospital, determined from the day of admission to the day of discharge. Theoretically, longer LOS (L-LOS) identifies more severe patients and/or

Address for correspondence: Dr. Umit Yasar Sinan, Instructor, Department of Cardiology, Istanbul University Institute of Cardiology, Haseki Street, 29/31, Istanbul, Turkey. E-mail: drumityasar@hotmail.com

This is an open access journal, and articles are distributed under the terms of the Creative Commons Attribution-NonCommercial-ShareAlike 4.0 License, which allows others to remix, tweak, and build upon the work non-commercially, as long as appropriate credit is given and the new creations are licensed under the identical terms.

For reprints contact: reprints@medknow.com

How to cite this article: Sinan UY, Erturk M, Yıldırım E, Koçyiğit D, Karaca I, Ertas F, et al. The predictors of long-term hospitalization in Turkish heart failure population: A subgroup analysis of journey heart failure-TR study: On behalf of journey heart failure-TR investigators. Int J Cardiovasc Acad 2018;4:82-5. 
complications occurring during hospitalization. It has been suggested a surrogate measure of hospital performance. ${ }^{[2,3]} \mathrm{LOS}$ is a key determinant of HF hospitalization costs. L-LOS is also associated with lower performance on quality of care measures and higher rates of subsequent readmission and mortality. Several studies revealed that possible determinants of L-LOS for HF patients include sociodemographic variables, medical comorbidity, disease severity (worse functional class and lower left ventricular ejection fraction [LVEF]), clinical presentation, inpatient treatment, inhospital progress, and development of iatrogenic complications. ${ }^{[3,4]}$ Some medical comorbidities that were known in prolonging LOS are concurrent stroke, worsening of renal function, atrial fibrillation, respiratory problems requiring specific treatment, and malnutrition. ${ }^{[5,6]}$

We aimed to determine the predictors of long-term hospitalization in Journey HF-TR population.

\section{Methods}

Journey-HF-TR study is a multicenter, cross-sectional, noninvasive, and observational study that was conducted in intensive care unit (ICU), CCU, and cardiology wards in seven geographical regions of Turkey. ${ }^{[7]}$ The patients hospitalized with the final diagnosis of AHF between September 2015 and 2016 were included in Journey-HF-TR study. According to LOS, patients were classified as patient with shorter LOS (S-LOS) (inhospital stay $<5$ days; S-LOS) and patients with L-LOS (inhospital stay $\geq 5$ days; L-LOS) in this subgroup analysis. Baseline and clinical characteristics, comorbidities, and laboratory data were collected during hospital admission. 12-lead electrocardiogram (ECG) and transthoracic echocardiography (TTE) were performed in all patients. Patients with systolic blood pressure higher than $140 \mathrm{mmHg}$ and diastolic blood pressure higher than $90 \mathrm{mmHg}$ or who were taking antihypertensive treatment were classified hypertensive (HT). Patients with fasting blood sugar higher than $125 \mathrm{mg} / \mathrm{dl}$ in two blood samples and glycated hemoglobin $\mathrm{A} 1 \mathrm{c}(\mathrm{HbA1c})$ higher than $6.5 \%$ or who were taking antidiabetic medication were classified as diabetes mellitus (DM). Anemia was defined as $\mathrm{Hb}$ level is $<13 \mathrm{~g} / \mathrm{dl}$ in men and $<12 \mathrm{~g} / \mathrm{dl}$ in women according to the World Health Organization criteria. Chronic kidney disease (CKD) was defined as if glomerular filtration rate (GFR) is $<60 \mathrm{ml} /$ per $\mathrm{min} / 1.73 \mathrm{~m}^{2}$. On TTE, patients were divided into three groups according to LVEF as patients with reduced EF (LVEF $<40 \%$ ), patients with midrange EF (LVEF: 40\%-49\%), and patients with preserved $\mathrm{EF}(\mathrm{LVEF}>50 \%)$.

\section{Statistical analysis}

Statistical analysis was performed using SPSS program (SPSS Inc., Chicago, IL, USA). Continuous variables are presented as the mean \pm standard deviation or median (minimum-maximum) if not normally distributed. Categorical variables are presented in frequency (percentages). Univariate analysis was performed to identify the variables associated with LOS. Those variables with $P<0.25$ at the univariate analysis were included in the multivariate model. A value of $P<0.05$ was considered to be statistically significant.

\section{RESULTS}

The study group was consisted of 1606 patients ( $57.2 \%$ male, mean age: $67.8 \pm 13.0$ years old). The initial functional capacity was New York Heart Association (NYHA) III-IV in $75.2 \%$ of patients. The median stay in CCU/ICU was 3 days, and total hospitalization duration was 7 days. The total inhospital mortality was $7.6 \%$ in the overall population.

One thousand and thirty-seven patients, whom inhospital stay duration were recorded in case report form, were included in this analysis. There were 487 patients (32.1\%) in S-LOS group and 1030 patients $(67.9 \%)$ in L-LOS group. Interestingly, the patients in S-LOS group were older than the patients in L-LOS group ( $69.1 \pm 13.0$ and $67.0 \pm 13.0$ years old, $P<0.05)$. There was much more male in L-LOS group $(55.6 \%$ and $44.4 \%$, $P<0.05)$.

The prevalence of HT, DM, AF, coronary artery disease, and anemia was similar between two groups. CKD was the unique comorbidity that was much more seen in patients with L-LOS than patients with S-LOS $(30.1 \%$ and $22.8 \%, P=0.003)$. The clinical presentation (de novo AHF or ADCHF) was similar between groups. Patients with L-LOS were much more presented with acute pulmonary edema (32.3\% and $28.3 \%)$, cardiogenic shock (4.1\% and $1.8 \%)$, and right ventricular failure (28.6\% and $20.7 \%)$ [Table 1 ].

Patients with L-LOS had much more severe disease. The prevalence of patients with advanced stage HF (NYHA III-IV) was higher in L-LOS group than S-LOS group (52. 3\% and $22.5 \%, P<0.001)$. Acute coronary syndrome (ACS)-related AHF was one of the contributing factors of long-term hospitalization. While the contributing factor was ACS in 16.3\% of patients with L-LOS, $11.7 \%$ of patients with S-LOS were hospitalized due to ACS-related AHF $(P<0.05)$. Hemodynamic monetarization (18.0\% and $11.9 \%)$, noninvasive $(18.3 \%$ and $10.9 \%$ ), and invasive ventilation ( $7.7 \%$ and $3.1 \%$ ) were the other causes of longer hospitalization ( $P<0.01$ for all three variable).

On physical examination, systolic blood pressure (130.3 \pm 29.9 and $127.1 \pm 30.9 \mathrm{mmHg})$, diastolic blood pressure $(82.4 \pm 12.2$ and $80.1 \pm 11.4 \mathrm{mmHg})$, and heart rate $(95.3 \pm 23.3$ and $93.2 \pm 23.5$ per min) were similar between S-LOS and L-LOS patient groups ( $P>0.05$ for all three variables). On ECG, QRS duration also was similar between two groups (106.6 \pm 25.9 and $105.7 \pm 40.8 \mathrm{msn} ; P<0.05$ ) [Table 2].

On laboratory, natriuretic peptide levels (N-terminal pro B-type natriuretic peptide) and GFR and $\mathrm{Hb}$ levels were also similar between two groups (respectively, $6701 \pm 978$ and $8129 \pm 188 \mathrm{pg} / \mathrm{ml}, 49.7 \pm 28.7$ and $49.4 \pm 31.4 \mathrm{ml} / \mathrm{min} / 1.73 \mathrm{~m}^{2}$, and $12.3 \pm 2.1$ and $12.1 \pm 2.1 \mathrm{~g} / \mathrm{dl} ; P>0.05$ for all three variables) [Table 2]. 
The mean EF was $35.5 \pm 12.9$ in S-LOS group patients, $32.1 \pm 12.1$ in L-LOS group patients $(P>0.05)$. While HF patients with preserved EF (HFpEF) was much more in patients with S-LOS $(21 \%$ and $16 \% ; P<0.001)$, HF patients with reduced EF (HFrEF) (64\% and 67\%) and HF patients with midrange EF (HFmrEF) (15\% and $17 \%)$ were similar between group $(P<0.05)$. There was no correlation between $\mathrm{EF}$ and L-LOS. As we know, HFpEF patients generally have more comorbidities and are older than other two groups. On the other hand, HFpEF patients have better systolic and diastolic BP than other EF groups. In advance stage, HF disease having lower BP is more problematic and causes L-LOS and higher mortality. Although we did not perform such statistical analysis, it may be the reason for S-LOS in HFpEF patients.

Univariate analysis showed correlation between LOS and male sex, advanced stage HF (NYHA III-IV), CKD, cardiogenic shock, pulmonary edema, right HF, invasive and noninvasive mechanical ventilation requirement, and hemodynamic monetarization requirement. In multivariate analysis, this correlation still was present for NYHA functional capacity, CKD, ACS-related HF, right HF, cardiogenic shock, invasive and noninvasive ventilation requirement, and hemodynamic monetarization requirement.

\section{Discussion}

In this study, we demonstrated that there was positive correlation between L-LOS and male sex, HF severity (NYHA III-IV), CKD, pulmonary edema, cardiogenic shock, right ventricular HF, HF related to ACS, invasive or noninvasive mechanical ventilation requirement, and hemodynamic monitor requirement. After multivariate analysis, this correlation remained constant for NYHA functional capacity, CKD, ACS-related HF, right HF, cardiogenic shock, invasive and noninvasive ventilation requirement, and hemodynamic monetarization requirement. Although the mean EF was similar between two groups, the prevalence of LOS was similar between patients with HFrEF and HFmrEF and had shorter in patients with HFpEF patients.

AHF is a life-threatening clinical syndrome that constitutes a majority of the hospitalizations among patients aged above 65 years. ${ }^{[1]}$ The LOS in AHF patients is a surrogate for the quality of health-care service. ${ }^{[2,3]}$ The L-LOS increases subsequent hospital readmission and mortality. It also increases health-care costs.

AHF patients with multiple comorbidities (HT, DM, AF, CKD, and anemia) have L-LOS ${ }^{[3,4]}$ Especially, anemia and worsening of renal failure during inhospital stay are possible determinants of L-LOS for AHF patients. ${ }^{[8-10]}$ Iron deficiency without the presence of anemia is an important consequence of HF. It causes worse prognosis and advanced stage disease in HF patients. ${ }^{[11]}$ In our study population, we did not check the patient's ferritin and transferrin saturation, but $\mathrm{Hb}$ levels and prevalence of anemia were similar in S-LOS and

\begin{tabular}{lccc}
\hline $\begin{array}{l}\text { Table 1: Demographical and clinical characteristics of } \\
\text { two groups }\end{array}$ \\
\hline Parameters & $\begin{array}{l}\text { L-LOS } \\
(\boldsymbol{n}=\mathbf{1 0 3 0})\end{array}$ & $\begin{array}{c}\text { S-LOS } \\
(\boldsymbol{n}=\mathbf{4 8 7})\end{array}$ & $\boldsymbol{P}$ \\
\hline Sex, male (\%) & 55.6 & 44.4 & $0.04^{*}$ \\
Age (years) & $67.0 \pm 13.0$ & $69.1 \pm 13.0$ & $0.03^{*}$ \\
NYHA III-IV (\%) & 52.3 & 22.5 & $<0.001$ \\
HT (\%) & 67.6 & 65.5 & 0.4 \\
DM (\%) & 43.0 & 40.7 & 0.3 \\
AF (\%) & 39.4 & 36.6 & 0.3 \\
Anemia (\%) & 57.2 & 54.4 & 0.5 \\
CKD (\%) & 30.1 & 22.8 & $0.003^{*}$ \\
Clinical presentation (\%) & & & \\
ADCHF & 81.7 & 79.5 & 0.2 \\
De novo AHF & 18.3 & 20.5 & 0.3 \\
APE & 32.3 & 28.3 & $0.04 *$ \\
CS & 4.1 & 1.8 & $0.04^{*}$ \\
RHF & 28.6 & 20.7 & $0.002^{*}$ \\
ACS & 16.3 & 11.7 & $0.04^{*}$ \\
Hemodynamic monitor & 18.0 & 11.9 & $<0.01$ \\
NIV & 18.3 & 10.9 & $<0.01$ \\
MV & 7.7 & 3.1 & $<0.01$ \\
\hline$* P<0.05$ is & & & \\
\hline
\end{tabular}

${ }^{*} P<0.05$ is significant. NYHA: New York Heart Association, HT: Hypertension, DM: Diabetes mellitus, AF: Atrial fibrillation, CKD: Chronic kidney disease, ADCHF: Acute decompensated chronic heart failure, AHF: Acute heart failure, ACS: Acute coronary syndrome, NIV: Noninvasive ventilation, APE: Acute pulmonary edema, CS: Cardiogenic shock, RHF: Right heart failure, MV: Mechanical ventilation, S-LOS: Shorter length of stay, L-LOS: Longer length of stay

Table 2: Physical examination, electrocardiogram, laboratory, and echocardiographic features of groups

\begin{tabular}{lccc}
\hline Parameters & L-LOS & S-LOS & $\boldsymbol{P}$ \\
\hline SBP $(\mathrm{mmHg})$ & $130.3 \pm 29.9$ & $127.1 \pm 30.9$ & 0.5 \\
DBP $(\mathrm{mmHg})$ & $82.4 \pm 12.2$ & $80.1 \pm 11.4$ & 0.5 \\
HR (bpm) & $95.3 \pm 23.3$ & $93.2 \pm 23.5$ & 0.9 \\
QRS duration (msn) & $106.6 \pm 25.9$ & $105.7 \pm 40.8$ & 0.06 \\
NT pro-BNP (pg/ml) & $6701 \pm 978$ & $8129 \pm 188$ & 0.4 \\
GFR (ml/pm/1.73 m²) & $49.7 \pm 28.7$ & $49.4 \pm 31.4$ & 0.06 \\
Hb (g/dl) & $12.3 \pm 2.1$ & $12.1 \pm 2.1$ & 0.3 \\
EF (\%) & $32.1 \pm 12.1$ & $35.5 \pm 12.9$ & 0.2 \\
EF groups (\%) & & & \\
HFrEF & 67 & 64 & 0.3 \\
HFmrEF & 17 & 15 & 0.3 \\
HFpEF & 16 & 21 & $<0.001$ \\
\hline
\end{tabular}

S-LOS: Shorter length of stay, L-LOS: Longer length of stay, SBP: Systolic blood pressure, DBP: Diastolic blood pressure, HR: Heart rate, NT-proBNP: N-terminal pro B-type natriuretic peptide, GFR: Glomerular filtration rate, Hb: Hemoglobin, EF: Ejection fraction, HF: Heart failure, HFrEF: HF patients with reduced EF, HFmrEF: HF patients with mid-range EF, HFpEF: HF patients with preserved EF

L-LOS patient's groups. The mean GFR value was below $60 \mathrm{ml} / \mathrm{min} / 1.73 \mathrm{~m}^{2}$. The prevalence of CKD patients was higher in L-LOS group. The prevalence of other comorbidities (HT, DM, AF, etc.) was similar between the two groups. ACS is an important clinical situation, especially for de novo AHF. Most of the patients with ACS-related AHF benefit from 
revascularization therapy. In our study group, ACS-related AHF caused longer hospital stay.

NYHA functional classification has been used to describe the severity of symptoms and exercise intolerance. The term advanced HF (NYHA III-IV) is used to characterize patients with severe symptoms, recurrent decompensation, and severe cardiac dysfunction. ${ }^{[1]}$ The prevalence of NYHA III-IV patients was higher in L-LOS group than S-LOS group. Cardiogenic shock and pulmonary edema are the two most serious clinical presentation of AHF. They increase LOS and inhospital mortality. We demonstrated correlation between L-LOS and pulmonary edema and cardiogenic shock, but after multivariate analysis, this correlation was just continued for cardiogenic shock. Right HF is an important consequence of HF patients. Right ventricular dysfunction predicted poor outcomes in patients with stable CHF. ${ }^{[12]}$ Yamin et al. showed right ventricular dysfunction as a predictor of longer hospital stay in patients with acute decompensated HF. ${ }^{[13]}$ Likewise, right $\mathrm{HF}$ was one of the determinants of L-LOS in patients with ADCHF in our study. Invasive or noninvasive mechanical ventilation is necessary for hypoxemic HF patients. It reflects the hypoxemia due to volume overload, especially in lung parenchyma. Hemodynamic monetarization is necessary for the management of inotropic or vasodilator therapy in patients with hypertensive or hypotensive HF. The requirement of respiratory support and hemodynamic monetarization was higher in L-LOS group than S-LOS group.

\section{Conclusion}

Our study is the largest scale study to investigate the determinants of longer hospital stay in Turkish HF population that was hospitalized due to AHF. It gives important clues to improve health-care quality and shorten the LOS in Turkey. L-LOS is an important surrogate subsequent hospital readmission and mortality. Since our study is retrospective in natüre, we do not have an idea about the prevalance of hospital readmission and mortality among patients with L-LOS. Prospective studies are necessary to present this relation.

Our study has several limitations. Surveys based on voluntary participation and recruitment of patients have obvious limitations that have to be acknowledged. Although participant sites were encouraged to enroll patients, as consecutively as possible, the study population may not represent the general population. The registry data are based on only by documentation of medical history, and management during hospitalization and the follow-up data are not obtained.

\section{Financial support and sponsorship} Nil.

\section{Conflicts of interest}

There are no conflicts of interest.

\section{References}

1. Go AS, Mozaffarian D, Roger VL, Benjamin EJ, Berry JD, Blaha MJ, et al. Heart disease and stroke statistics-2014 update. Circulation 2014;129:e28-92.

2. Heidenreich PA, Sahay A, Kapoor JR, Pham MX, Massie B. Divergent trends in survival and readmission following a hospitalization for heart failure in the veterans affairs health care system 2002 to 2006. J Am Coll Cardiol 2010;56:362-8.

3. Cotter G, Davison BA, Milo O, Bourge RC, Cleland JG, Jondeau G, et al. Predictors and association with outcomes of length of hospital stay in patients with acute heart failure: Results from VERITAS. J Card Fail 2016;22:815-22.

4. Wright SP, Verouhis D, Gamble G, Swedberg K, Sharpe N, Doughty RN, et al. Factors influencing the length of hospital stay of patients with heart failure. Eur J Heart Fail 2003;5:201-9.

5. Aziz EF, Javed F, Pratap B, Musat D, Nader A, Plumi S, et al. Malnutrition as assessed by nutritional risk index is associated with worse outcome in patients admitted with acute decompensated heart failure: An ACAP-HF data analysis. Heart Int 2011;6:e2.

6. Kyle UG, Schneider SM, Pirlich M, Lochs H, Hebuterne X, Pichard C. Does nutritional risk, as assessed by nutritional risk index, increase during hospital stay? A multi-national population based study. Clin Nutr 2005;24:516-24.

7. Sinan UY, Ekmekçi A, Özbay B, Akçay FA, Bekar L, Koza Y, et al. The real life data of hospitalized patients with heart failure: On behalf of journey HF-TR study investigators. Anatol J cardiol 2018. Doi:10.14744/Anatol J Cardiol.2018.50880.

8. Caughey MC, Avery CL, Ni H, Solomon SD, Matsushita K, Wruck LM, et al. Outcomes of patients with anemia and acute decompensated heart failure with preserved versus reduced ejection fraction (from the ARIC study community surveillance). Am J Cardiol 2014;114:1850-4.

9. Shirakabe A, Hata N, Kobayashi N, Shinada T, Tomita K, Tsurumi M, et al. Prognostic impact of acute kidney injury in patients with acute decompensated heart failure. Circ J 2013;77:687-96.

10. Reynolds K, Butler MG, Kimes TM, Rosales AG, Chan W, Nichols GA. Relation of acute heart failure hospital length of stay to subsequent readmission and all-cause mortality. Am J Cardiol 2015;116:400-5.

11. Ponikowski P, Voors AA, Anker SD, Bueno H, Cleland JG, Coats AJ, et al. 2016 ESC guidelines for the diagnosis and treatment of acute and chronic heart failure: The task force for the diagnosis and treatment of acute and chronic heart failure of the European society of cardiology (ESC). Developed with the special contribution of the heart failure association (HFA) of the ESC. Eur J Heart Fail 2016;18:891-975.

12. Gajanana D, Seetha Rammohan H, Alli O, Romero-Corral A, Purushottam B, Ponamgi S, et al. Tricuspid annular plane systolic excursion and its association with mortality in critically ill patients. Echocardiography 2015;32:1222-7.

13. Yamin PP, Rharjo SB, Putri VK, Hersunarti N. Right ventricular dysfunction as predictor of longer hospital stay in patients with acute decompensated heart failure: A prospective study in Indonesian population. Cardiovasc Ultrasound 2016;14:14-25. 